\title{
Gewicht- und Volummessung der nicht mazerierten langen Extremitäts- knochen der Japaner.
}

\author{
Von
}

Kenzo Matsui.

Aus dem Anatomischen Institut der Med. Fakultät Okayama

(Vorstand: Prof. M. Seki).

Die Gewichtsmessungen der Knochen wurden in anthropologischer Hinsicht nur selten vorgenommen. In diesen Fällen beschränkten sich die Messungen meist auf mazerierten ausgetrockneten Knochen. Angaben über das Gewicht der nicht mazerierten Knochen finden sich kaum in der Literatur. Ich habe das Gewicht der nicht mazerierten, in Formalin fixierten langen Extremitätsknochen der Japaner gemessen, daneben wurde das Volumen einiger Knochen bestimmt, dessen Wert mit demjenigen des Gewichtes vergleichend studiert wurde.

\section{Material und Methode.}

Es wurden ter Humerus, die Ulna, der Radius, das Femur und die Tibia aus 65 männlichen und 22 weiblichen Leichen, die durch die Injektion der Blutgefässe mit Formalin fixiert wurden, ausgenommen und von allen Weichteilen, mit Ausnahme der Knochenhaut und des Gelenkknorpels, wie von Gelenkkapsel, Bändern, Muskelsehnen usw., sorgfältig befreit und gewogen. Die Volummenssungen wurden nach Eintauchen der Knochen in das Wasser in einem Zylinder durch die Messung der verdrängten Wassermenge vorgenommen.

Das Gewicht wurde immer an den beiderseitigen Knochen von einem Individuum gemessen. Bei den männlichen Leichen wurden Humerus, Ulna und Radius von 63, Femur von 60 und Tibia von 65 Individuen in Betracht gezogen, bei den weiblichen Humerus von 21, Ulna, Radius, Femur und Tibia von 22 Individuen. Die Volummessungen fanden dagegen nur an den Knochen aus männliehen Leichen statt, und zwar an Humerus, Ulna, und Radius von 8 und Femur und Tibia von 9 Individuen. 


\section{Ergebnisse und Auswertung.}

\section{A. Gewichtmessung.}

1. Die Verteilung der Häufigkeit der Gewichte der Knochen sind wie in Tabelle 1-4. Weil die Knochen der Männer meist viel schwerer sind als die der Frauen, ist die Variationsbreite der Knochengewichte beider Geschlechter beträchtlich gross.

2. Der Unterschied in Gewicht der Knochen nach dem Geschlecht und dem Körperseiten zwischen rechts und links sind in Tabelle 4 angegeben.

Tabelle 1. Verteilung der Häufigkeit der Humerusgewichte.

\begin{tabular}{|c|c|c|c|}
\hline & \multicolumn{1}{|c|}{$\delta$} & $\wp$ & $\delta+q$ \\
\cline { 2 - 4 } & r. 63 & r. 21 & r. 81. \\
\hline $95-110$ & 1.63 & 1.21 & 1.84 \\
\hline $110-125$ & & 3 & 3 \\
$125-140$ & & 14 & 14 \\
$140-155$ & 1 & 9 & 10 \\
$155-170$ & 7 & 12 & 19 \\
$170-185$ & 12 & 0 & 12 \\
$185-200$ & 22 & 0 & 22 \\
$200-215$ & 22 & 3 & 25 \\
$215-230$ & 30 & 1 & 31 \\
$230-245$ & 17 & & 17 \\
$245-260$ & 9 & & 9 \\
$260-275$ & 4 & & 4 \\
\hline
\end{tabular}


Gewicht- und Volummessung der nicht mazerierten langen Extremitätsknochen. 233 Tabelle 2. Verteilung der Häufigkeit der Ulna- und Radiusgewichte.

\begin{tabular}{|c|c|c|c|c|c|c|}
\hline \multirow{4}{*}{ Gewicht (g) } & \multicolumn{3}{|c|}{ Ulna } & \multicolumn{3}{|c|}{ Radius } \\
\hline & $\hat{\jmath}$ & ? & $\delta+o$ & $\hat{\delta}$ & ? & $\hat{o}+q$ \\
\hline & r. 63 & r. 22 & r. 85 & r. 63 & r. 22 & r. 85 \\
\hline & I. 63 & 1. 22 & 1. 85 & 1. 63 & 1. 22 & I. 85 \\
\hline $20-25$ & & & & & 1 & 1 \\
\hline $25-30$ & & 1 & 1 & & 3 & 3 \\
\hline $30-35$ & 3 & 21 & 21 & & 3 & 3 \\
\hline $35-40$ & 6 & 8 & 14 & & 13 & 13 \\
\hline $40-45$ & 7 & 7 & 14 & 4 & 11 & 15 \\
\hline $45-50$ & 21 & 0 & 21 & 5 & 9 & 14 \\
\hline $50-55$ & 24 & 4 & 28 & 6 & 3 & 9 \\
\hline $55-60$ & 26 & & 26 & 20 & 1 & 21 \\
\hline $60-65$ & 16 & & 16 & 24 & 3 & 27 \\
\hline $65-70$ & 17 & & 17 & 15 & & 15 \\
\hline $70-75$ & 6 & & 6 & 16 & & 16 \\
\hline $75-80$ & & & & 24 & & 24 \\
\hline 8085 & & & & 8 & & 8 \\
\hline $85-90$ & & & & 4 & & 4 \\
\hline
\end{tabular}

Tabelle 3. Verteilung der Häufigkeit der Femur- und Tibiagewichte.

\begin{tabular}{|c|c|c|c|c|c|c|}
\hline & \multicolumn{3}{|c|}{ Femur } & \multicolumn{3}{c|}{ Tibia } \\
\cline { 2 - 7 } Gewicht (g) & $\delta$ & $\delta$ & $\delta+7$ & $\delta$ & $\wp$ & $\delta+\gamma$ \\
\cline { 2 - 7 } & r. 60 & r. 22 & r. 82 & r. 65 & r. 22 & r. 87 \\
& 1.60 & 1.22 & 1.82 & 1.65 & 1.22 & 1.87 \\
\hline $100-150$ & & & & & 1 & 1 \\
$150-200$ & & & & 2 & 4 & 6 \\
$200-250$ & & & & 5 & 24 & 29 \\
$250-300$ & & 3 & 3 & 29 & 12 & 41 \\
$300-350$ & 2 & 2 & 4 & 50 & 3 & 53 \\
$350-400$ & 0 & 12 & 12 & 30 & & 30 \\
$400-450$ & 10 & 15 & 25 & 11 & & 11 \\
$450-500$ & 11 & 6 & 17 & 2 & & 2 \\
$500-550$ & 27 & 4 & 31 & & & \\
$550-600$ & 31 & 2 & 33 & & & \\
$600-650$ & 25 & & 25 & & & \\
$650-700$ & 9 & & 9 & & & \\
$700-750$ & 3 & & 3 & & & \\
$750-800$ & 2 & & 2 & & & \\
\hline
\end{tabular}


Tabelle 4. Unterschied in Gewicht der Knochen nach dem Geschlecht und den Körperseiten zwischen rechts und links

(Mittelwert und wahrscheinlicher Fehler).

\begin{tabular}{|c|c|c|c|c|c|c|c|c|c|c|}
\hline & \multicolumn{3}{|c|}{$\delta$} & \multicolumn{3}{|c|}{ ㅇ } & \multicolumn{3}{|c|}{$\delta+q$} \\
\hline & & n & $\mathbf{M} \pm \mathbf{E}(\mathbf{M})$ & Max-Min & $\mathbf{n}$ & $\mathrm{M} \pm \mathrm{E}(\mathrm{M})$ & Max-Min & $n$ & $M \pm E(M)$ & Max-Min \\
\hline \multirow{2}{*}{\multicolumn{2}{|c|}{ Humerus ${ }_{1 .}^{r}$}} & 63 & $201 \pm 2.7$ & $262-140$ & 21 & $137 \pm 3.0$ & $205-103$ & 84 & $185-2.8$ & $262-103$ \\
\hline & & 63 & $206 \pm 2.1$ & 253-135 & 21 & $135 \pm 3.0$ & 194-102 & 84 & $186-2.8$ & $253-102$ \\
\hline \multirow{2}{*}{ Ulna } & r. & 63 & $67 \pm 0.9$ & $88-44$ & 22 & $44 \pm 1.1$ & $64-31$ & 85 & $6 \mathrm{I} \pm 1.0$ & $88-31$ \\
\hline & 1. & 63 & $66 \pm 0.9$ & $84-41$ & 22 & $43 \pm 1.0$ & 63- 29 & 85 & $60 \pm 1.0$ & $84-29$ \\
\hline \multirow{2}{*}{ Radius } & r. & 63 & $56 \pm 0.8$ & $74-35$ & 22 & $37 \pm 0.9$ & $54-28$ & 85 & $51 \pm 0.9$ & $74-28$ \\
\hline & 1. & 63 & $55 \pm 0.8$ & $72-32$ & 22 & $36 \pm 1.0$ & $53-25$ & 85 & $50 \pm 0.9$ & $72-25$ \\
\hline \multirow{2}{*}{ Femur } & r. & 60 & $563 \pm 7.0$ & 777-309 & 22 & $411 \pm 10.0$ & $590-266$ & 82 & $522 \pm 7.6$ & $777-266$ \\
\hline & 1. & 60 & $563 \pm 6.9$ & $782-327$ & 22 & $427 \pm 9.6$ & 596-285 & 82 & $527 \pm 7.2$ & $732-285$ \\
\hline \multirow{2}{*}{ Tibia } & r. & 65 & $333 \pm 4.8$ & $507-170$ & 22 & $236 \pm 6.0$ & $348-143$ & 87 & $308 \pm 4.9$ & $507-143$ \\
\hline & 1. & 65 & $330 \pm 4.8$ & $492-167$ & 22 & $241 \pm 5.5$ & 246-163 & 87 & $308 \pm 4.7$ & $492-163$ \\
\hline
\end{tabular}

3. Die Häufigkeit der Fälle, bei denen die Knochen rechts bzw. links schwerer sind, sieht man in Tabelle 5.

Tabelle 5. Häufigkeit der Fälle, bei denen die Knochen rechts bzw. links schwerer sind.

\begin{tabular}{|c|c|c|c|c|c|c|c|c|c|}
\hline & \multicolumn{3}{|c|}{$\delta$} & \multicolumn{3}{|c|}{ 우 } & \multicolumn{3}{|c|}{$\delta+q$} \\
\hline & r. $>1$ & r. $=1$. & r. $<1$. & r. $>$ l. & r. $=1$. & r. $<1$. & r. $>1$ & $\mathbf{r .}=1$. & r. $<1$. \\
\hline Humerus & $\begin{array}{c}47 \\
(74.6 \%)\end{array}$ & $\begin{array}{c}1 \\
(1.6 \%)\end{array}$ & $\begin{array}{c}15 \\
(23.8 \%)\end{array}$ & $\begin{array}{c}11 \\
(52.4 \%)\end{array}$ & $\begin{array}{c}3 \\
(14.3 \%)\end{array}$ & $\begin{array}{c}7 \\
(33.3 \%)\end{array}$ & $\begin{array}{c}58 \\
(69.0 \%)\end{array}$ & $\begin{array}{c}4 \\
(4.8 \%)\end{array}$ & $\mid \begin{array}{c}22 \\
(26.2 \%)\end{array}$ \\
\hline Ulna & $\begin{array}{c}37 \\
(58.7 \%)\end{array}$ & $\begin{array}{c}5 \\
(7.9 \%)\end{array}$ & $\begin{array}{c}21 \\
(33.3 \%)\end{array}$ & $\begin{array}{c}11 \\
(50.0 \%)\end{array}$ & $\begin{array}{c}2 \\
(9.1 \%)\end{array}$ & $\begin{array}{c}9 \\
(40.9 \%)\end{array}$ & $\begin{array}{c}48 \\
(56.3 \%)\end{array}$ & $\begin{array}{c}7 \\
(8.2 \%)\end{array}$ & $\begin{array}{c}30 \\
(35.2 \%)\end{array}$ \\
\hline Radius & $\begin{array}{c}36 \\
(57.1 \%)\end{array}$ & $\begin{array}{c}11 \\
(17.5 \%)\end{array}$ & $\begin{array}{c}16 \\
(25.4 \%)\end{array}$ & $\begin{array}{c}13 \\
(59.1 \%)\end{array}$ & $\begin{array}{c}5 \\
(22.7 \%)\end{array}$ & $\begin{array}{c}4 \\
(18.1 \%)\end{array}$ & $\begin{array}{c}49 \\
(57.6 \%)\end{array}$ & $\begin{array}{c}16 \\
(18.8 \%)\end{array}$ & $\begin{array}{c}20 \\
(23.5 \%)\end{array}$ \\
\hline Femur & $\begin{array}{c}27 \\
(45.0 \%)\end{array}$ & 0 & $\begin{array}{c}33 \\
(55.0 \%)\end{array}$ & $\begin{array}{c}8 \\
(36.4 \%)\end{array}$ & $\begin{array}{c}1 \\
(4.5 \%)\end{array}$ & $\begin{array}{c}13 \\
(59.1 \%)\end{array}$ & $\begin{array}{c}35 \\
(42.7 \%)\end{array}$ & $\left(\begin{array}{c}1 \\
(1.2 \%)\end{array}\right.$ & $\mid \begin{array}{c}46 \\
(56.1 \%)\end{array}$ \\
\hline Tibia & $\begin{array}{c}33 \\
(50.8 \%)\end{array}$ & 0 & $\begin{array}{c}32 \\
(49.2 \%)\end{array}$ & $\begin{array}{c}12 \\
(54.5 \%)\end{array}$ & $\begin{array}{c}1 \\
(4.5 \%)\end{array}$ & $\begin{array}{c}9 \\
(40.9 \%)\end{array}$ & $\begin{array}{c}45 \\
(51.7 \%)\end{array}$ & $\begin{array}{c}1 \\
(1.1 \%)\end{array}$ & $\mid \begin{array}{c}41 \\
(47.1 \%)\end{array}$ \\
\hline
\end{tabular}


Gewicht- und Volummessung der nicht mazerierten langen Extremitätsknochen. 235

Aus der Tabelle ersieht man, dass die oberen Extremitätsknochen durchschnittlich immer rechts schwerer wiegen als links, dass aber dies von den unteren Extremitätsknochen nicht gilt. Das Femur ist vielmehr durchschnittlich auf der linken Seit schwerer.

4. Zum Schluss vergleicht man das Gewicht unseren nicht mazerierten Materials mit demjenigen der nach Mazeration getrockneten Knochen aus Nordjapan $(\delta+\%, \mathrm{n}=50)$ nach Nunoda (1949) und aus Ostjapan ( $\hat{o}+\uparrow, \mathrm{n}=138$ ) nach Nagura (1932). Die mazerierten Knochen sind vielleicht nicht in Benzin oder dgl. entfettet. Sie sind doch durch die Fäule in Wasser an einem warmen Ort mehr oder weniger stark ihrer organischen Substanz beraubt. In Tabelle 7 sind die Verminderungen des Gewichtes der Knochen in Prozent, berechnet aus den in Tabelle 6 angegebenen Daten, gezeigt.

Tabelle 6. Vergleich des Gewichtes der nicht mazerierten und der nach Mazeration getrockneten Knochen.

\begin{tabular}{|c|c|c|c|c|c|c|c|}
\hline & \multicolumn{2}{|c|}{ Nach Matsui } & \multicolumn{2}{|c|}{ Nach Nunoda } & \multicolumn{2}{|c|}{ Nach Nagura } \\
\hline & & $M \pm E$ (M) & Max-Min & $\mathrm{M} \pm \mathrm{E}(\mathrm{M})$ & Max-Min & $\mathrm{M} \pm \mathrm{E}(\mathrm{M})$ & Max-Min \\
\hline \multirow{2}{*}{\multicolumn{2}{|c|}{ Humerus ${ }_{1 .}^{r}$}} & $185 \pm 2.8$ & $262-103$ & $97 \pm 2.7$ & $190-46$ & $98 \pm 1.5$ & $173-45$ \\
\hline & & $186 \pm 2.8$ & $253-102$ & $96 \pm 2.9$ & $192-45$ & $95 \pm 1.5$ & $164-42$ \\
\hline \multirow{2}{*}{ Ulna } & r. & $61 \pm 1.0$ & $88-31$ & $37 \pm 1.2$ & $71-15$ & $40 \pm 0.6$ & $61-17$ \\
\hline & 1. & $60 \pm 1.0$ & $84-29$ & $37 \pm 1.2$ & $70-15$ & $39 \pm 0.6$ & $61-18$ \\
\hline \multirow{2}{*}{ Radius } & r. & $51 \pm 0.9$ & $74-28$ & $31 \pm 1.1$ & $57-15$ & $31 \pm 0.5$ & $50-14$ \\
\hline & 1. & $50 \pm 0.9$ & $72-25$ & $31 \pm 1.1$ & $58-14$ & $31 \pm 0.5$ & $51-13$ \\
\hline \multirow{2}{*}{ Femur } & r. & $522 \pm 7.6$ & $777-266$ & $271 \pm 8.4$ & $545-125$ & $268 \pm 3.9$ & $416-145$ \\
\hline & 1. & $527 \pm 7.2$ & $782-285$ & $272 \pm 8.4$ & $545-127$ & $270 \pm 3.8$ & $432 \cdot 122$ \\
\hline \multirow{2}{*}{ Tibia } & r. & $308 \pm 4.9$ & $507-143$ & $156 \pm 5.4$ & $315-68$ & $153 \pm 2.4$ & 244- 64 \\
\hline & 1. & $308 \pm 4.7$ & $492-163$ & $155 \pm 5.3$ & $317-66$ & $152 \pm 2.4$ & $244-64$ \\
\hline
\end{tabular}


Tabelle 7. Verlust der Knochen an Gewicht nach der Mazeration und Ausstrocknung in Prozent.

\begin{tabular}{|cc|c|}
\hline Humerus & r. & $47.2 \%$ \\
& 1. & $48.5 \%$ \\
\hline Ulna & r. & $37.7 \%$ \\
& 1. & $37.6 \%$ \\
\hline \multirow{2}{*}{ Radius } & r. & $38.2 \%$ \\
& 1. & $38.0 \%$ \\
\hline \multirow{2}{*}{ Femur } & r. & $48.3 \%$ \\
& 1. & $48.5 \%$ \\
\hline Tibia & r. & $50.0 \%$ \\
\hline
\end{tabular}

Man ersieht, dass die Tibia am grössten ihr Gewicht verliert (um $50 \%$ ), die Ulna aber am kleinsten (um 38\%).

\section{B. Volummessung.}

Die Volummessung geschah nur an wenigen männlichen Knochen (Tabelle 8). Das Gewicht der Knochen pro ccm findet sich in Tabelle 9.

Tabelle 8. Volumen der Knochen $(\delta)$

\begin{tabular}{|cc|c|c|c|}
\hline & & $\mathrm{n}$ & $\mathrm{M}$ & $\mathrm{Max}-\mathrm{Min}$ \\
\hline Humerus & r. & 8 & 151 & $182-130$ \\
& 1. & 8 & 147 & $176-130$ \\
\hline \multirow{2}{*}{ Ulna } & r. & 8 & 47 & $58-37$ \\
& 1. & 8 & 46 & $56-38$ \\
\hline \multirow{2}{*}{ Radius } & r. & 8 & 42 & $52-33$ \\
& 1. & 8 & 40 & $50-33$ \\
\hline \multirow{2}{*}{ Femur } & r. & 8 & 440 & $495-360$ \\
& 1. & 8 & 439 & $500-380$ \\
\hline \multirow{2}{*}{ Tibia } & r. & 8 & 269 & $310-222$ \\
& l. & 8 & 290 & $330-218$ \\
\hline
\end{tabular}

Tabelle 9. Spezifisches Gewicht der männlichen, nicht mazerierten Knochen.

\begin{tabular}{|cc|c|}
\hline Humerus & r. & 1.33 \\
& 1. & 1.40 \\
\hline Ulna & r. & 1.44 \\
& 1. & 1.44 \\
\hline Radius & r. & 1.32 \\
& I. & 1.36 \\
\hline \multirow{2}{*}{ Femur } & r. & 1.27 \\
& l. & 1.28 \\
\hline Tibia & r. & 1.23 \\
& 1. & 1.13 \\
\hline
\end{tabular}


Gewicht- und Volummessung der nicht mazerierten langen Extremitätsknochen. 237

Das spezifische Gewicht der Ulna ist am grössten, und dasjenige der Tibia am kleinsten. $\mathrm{Zu}$ bemerken ist, dass die Ulna, wie oben erwähnt, durch die Mazeration ihre Substanz relativ am wenigsten verliert, dagegen die Tibia dabei am grössten.

\section{Zusammenfassung.}

1. Die nicht mazerierten grossen langen Extremitätsknochen aus 65 männlichen und 22 weiblichen Leichen wurden untersucht. Dieselben waren bei dem männlichen Geschlecht meist viel schwerer als bei dem weiblichen. Der Mittelwert des Knochengewichtes war bei dem männlichen Geschlecht: Humerus r. $201 \mathrm{~g}, 1.206 \mathrm{~g}$, Ulna r. $67 \mathrm{~g}, 1.66 \mathrm{~g}$, Radius r. 56 g, 1. 55 g, Femur r. $563 \mathrm{~g}, 1.563 \mathrm{~g}$, Tibia r. $333 \mathrm{~g}, 1.330 \mathrm{~g}$, uud bei dem weiblichen: Humerus r. $137 \mathrm{~g}, 1.135 \mathrm{~g}$, Ulna r. $44 \mathrm{~g}, 1$. $43 \mathrm{~g}$, Radius r. $37 \mathrm{~g}, 1.36 \mathrm{~g}$, Femur r. $411 \mathrm{~g}, 1.427 \mathrm{~g}$, Tibia r. $236 \mathrm{~g}, \mathrm{l}$. $241 \mathrm{~g}$.

2. Die oberen Extremitätsknochen waren in überwiegender Mehrzahl der Fälle rechts schwerer als links. Bei den unteren Extremitätsknochen lagen jedoch die Verhältnisse anderes, und das linke Femur erweist sich in der Überzahl der Fälle als schwerer als das rechte.

3. Durch die Mazeration verliert unter den hier' untersuchten Knochen die Tibia am grössten ihre Substanz (r. um $50.0 \%$, 1. um $50.0 \%$ ), und die Ulna am wenigsten (r. um $37.7 \%$, l. um $37.6 \%$ ).

4. Das spezifische Gewicht der männlichen Knochen war an der Ulna am grössten (r. 1.44, 1. 1.44) und an der Tibia am kleinsten (r. 1.23, 1. 1.13).

\section{Literaturverzeichnis.}

1) Davida, E.: Proportionsuntersuchungen auf Grund des Knochenvolumens und der Volumenindex der Extremitätsknochen. Anat. Anz. 61 (1926).

2) In a ba, F.: Über die symmetrischen Verhältnisse der Messungswerte der Extremitätsknochen. (Jap.) Nichidai I.Z. 7 (1943).

3) Matsushima, H.: Über das Gewicht der Knochen der männlichen Japaner. (Jap.) Acta med. 2 (1928).

4) Nagura, S.: Pri la pesa interrilato de la longaj ostoj de membroj. (Jap.) Tokyo Igakkai Z. 46 (1932).

5) Nunoda, T.: Über die Gewichtsproportion der Extremitätsknochen der Japaner in Nordjapan. (Jap.) Arb. Anat. Inst. Kanazawa 37 (1949).

6) Saito, Y.: Über das Gewicht der oberen Extremitätsknochen der Japaner. (Jap.) Arb. Anat. Inst. Kanazawa 17 (1934).

7) Ueda, Ts. : Über die Rechnungsmethode des Standardfehlers, wahrscheinlichen Fehlers usw. bei der Rechts.Links-Messung. (Jap.) Kaibo Z. 4 (1931). 